\title{
Corticotropin-Releasing Factor in the Hippocampus: Eustress or Distress?
}

\author{
Christopher M. Stern \\ Department of Neuroscience and Graduate Program in Neuroscience, University of Minnesota, Minneapolis, Minnesota 55455 \\ Review of Ivy et al.
}

Corticotropin-releasing factor/hormone (CRF) was initially characterized as an upstream mediator of the acute stress response. Presentation of a stressful stimulus results in activation of CRF-secreting neurons of the paraventricular nucleus of the hypothalamus. This leads to the rapid downstream secretion of corticosteroids that, in part, characterize the acute stress response, which organisms depend upon for survival. In addition to facilitating the appropriate behavioral response to a noxious stimulus, stress may also improve adaptive responses to similar subsequent stressful stimuli by facilitating memory. For instance, brief bouts of acute stress in rodents have been shown to facilitate learning and memory, a phenomenon dependent on extrahypothalamic brain regions such as the hippocampus (Radulovic et al., 1999).

In addition to these favorable consequences, stress also detrimentally influences organismal physiology. Excessive early life and chronic stress can lead to depression, anxiety, cognitive detriments, and memory impairment later in life (McEwen, 1999). Although glucocorticoids have been thought of as the primary mediators of stress-induced modulation

Received 0ct. 26, 2010; revised Nov. 22, 2010; accepted Dec. 1, 2010.

I thank Jessie Luoma and Dr. Paul Mermelstein for helpful discussion on this manuscript.

Correspondence should be addressed to Christopher M. Stern, Department of Neuroscience, University of Minnesota, 6-145 Jackson Hall, 321 Church Street SE, Minneapolis, MN 55455. E-mail:christopher.stern@gmail.com. DOI:10.1523/JNEUROSC1.5611-10.2011

Copyright $\odot 2011$ the authors $\quad 0270-6474 / 11 / 311935-02 \$ 15.00 / 0$ of hippocampal function, recent evidence suggests that direct actions of CRF in the hippocampus play a crucial role in these effects as well. In fact, an emerging hypothesis from studies of stress influence on hippocampal function is that stress and CRF can exert bidirectional effects depending on a variety of factors including stress type, intensity, duration, and physiological parameter. For instance, acute injection of CRF 5 min before fear conditioning enhances subsequent performance on this hippocampal-dependent task (Radulovic et al., 1999) and a brief application ( $<20 \mathrm{~min}$ ) of CRF to hippocampal slices facilitates hippocampal long-term potentiation (LTP) (Blank et al., 2002), whereas stress of longer duration ( $\geq 1 \mathrm{~h}$ ) or sustained CRF application impairs hippocampal LTP (Rebaudo et al., 2001) and hippocampal-dependent learning and memory (Chen et al., 2010).

In a recent article in The Journal of Neuroscience, Ivy et al. (2010) add to this growing literature by characterizing the detrimental effects of chronic early life stress (CES) on adult hippocampal function. They used a well established chronic stress model, in which a rat dam and its litter of 2-d-old (P2) pups were placed in a cage with plastic-coated aluminum mesh bottom devoid of bedding material. After 1 week, the dam and litter were moved back into a cage with nesting chips. The pups were allowed to mature normally before the effects of stress were assayed. Ivy et al. (2010) measured stress effects on dendritic spines, hippocampal physiology, and hippocampal-dependent behavior.

CES resulted in CA1 pyramidal neuron dendrite atrophy. Reductions were greatest in proximal stratum radiatum, which receives synaptic input from Schaffer collaterals. Additionally, total dendritic length was significantly reduced in CES rats relative to control animals. These effects were blocked by treatment of rat pups with a CRF receptor (CRFR) 1 antagonist immediately after CES, demonstrating the necessity of CRFR1 activation in this effect. Ivy et al. (2010) ruled out the possibility that the antagonist was acting by influencing CRF-induced glucocorticoid release by showing the CRFR1 antagonist had no effect on circulating glucocorticoid levels. Importantly, a similar effect was produced by incubation of hippocampal organotypic slice cultures from P1 mice with CRF for 2 weeks.

Since dendritic spines are a primary site of excitatory synaptic transmission in the hippocampus, stress- and CRFinduced reductions in dendritic morphology could have detrimental affects on excitatory neurotransmission. To test this, Ivy et al. (2010) assayed synaptic plasticity in the CA3-CA1 apical dendritic synapses (Schaffer collateral pathway), which are detrimentally affected by early life stress (McEwen, 1999). CES had no effect on baseline synaptic transmission as measured by field EPSPs (fEPSPs). High-frequency stimulation significantly potentiated fEPSPs in slices from control animals, but potentiation was significantly attenuated in slices 
from CES animals. CES-induced attenuation was blocked by post-CES treatment with the CRFR1 antagonist, suggesting a role for CRFR1 in stress-induced attenuation of synaptic plasticity. Thus, Ivy et al. (2010) show a physiological correlate of the observed derangement in dendritic morphology.

Importantly, the authors went on to demonstrate that there are behavioral parallels of the demonstrated deficits in hippocampal physiology and morphology. CES animals showed impaired hippocampus-dependent spatial memory in both the Morris water maze and novel object recognition tasks. These CESinduced deficiencies were similarly prevented by treatment with the CRFR1 antagonist.

Since the CRFR1 antagonist prevented CES-induced deficits in hippocampal function independent of any effects on corticosteroid levels, Ivy et al. (2010) concluded that the antagonism must be at the level of central CRFR1 as opposed to an effect on peripheral corticosteroid synthesis and secretion. What, then, is the source of hippocampal CRF that is mediating these effects? Previous work from the same laboratory showed that basket-cell interneurons in the pyramidal layer of the hippocampus express CRF, and that CRF is released in the hippocampus in response to stress (Chen et al., 2004). Furthermore, CRFRs are localized to dendritic spines of pyramidal neurons (Chen et al., 2004). These data are in agreement with other studies demonstrating both CRF (De Souza et al., 1985) and CRFR (Merchenthaler, 1984) expression in the hippocampus. Together, these data suggest that CRF is an endogenous neurotransmitter and/or neuromodulator that is released within the hippocampus in response to stress.

Although Ivy et al. (2010) do not directly test this hypothesis, they provide strong correlative evidence that intrahippocampal CRF neurotransmission is affected by CES. Specifically, Ivy et al. (2010) demonstrated that the number of
CRF-positive interneurons in CA1 and CA3 hippocampal subregions was elevated in middle-aged rats that had undergone CES, suggesting that CES induced upregulation of CRF expression in these neurons. Nonetheless, it is important to determine whether increased hippocampal CRF expression (and putative release) is causally involved in CESmediated deficits.

The molecular mechanisms by which locally released CRF might influence longterm changes in hippocampal function have yet to be definitively determined. Ivy et al. (2010) reported that CES effects resulted from central CRFR1 activation. Given that CRF and CRFR1 activation can initiate a plethora of downstream intracellular signaling pathways across distinct cell populations (Hillhouse and Grammatopoulos, 2006), it is important to determine the intracellular signaling pathways that underlie CES influence of hippocampal function. In light of the fact that CES resulted in deranged dendritic spine dynamics and LTP expression, it will behoove future investigators to focus on signaling molecules that have been implicated in these phenomena.

In summary, this work by Ivy et al. (2010) adds to a growing body of literature describing the profound influence that stress peptides exert on hippocampal physiology and function. Whereas much past focus has been placed on the effects of corticosteroids (McEwen, 1999), this report supports the notion that CRF plays a direct role in mediating the effects of stress in the hippocampus. A second emerging theme is that stress and CRF can exert bidirectional influences on the hippocampus, with type, intensity, and duration of stress influencing the direction of the effect. Future experiments should be aimed at determining what specific factors determine the direction of the stress effect. The results from Ivy et al. (2010) support the notion that exposure to chronic stress early in life can have permanent and lasting detrimental effects on physiology and behavior. A thorough un- derstanding of the specific cellular and molecular mechanisms that underlie the bidirectional effects of stress and CRF influence of hippocampus will be required for comprehensive understanding of the extrahypothalamic stress response.

\section{References}

Blank T, Nijholt I, Eckart K, Spiess J (2002) Priming of long-term potentiation in mouse hippocampus by corticotropin-releasing factor and acute stress: implications for hippocampusdependent learning. J Neurosci 22:3788-3794.

Chen Y, Brunson KL, Adelmann G, Bender RA, Frotscher M, Baram TZ (2004) Hippocampal corticotropin releasing hormone: pre- and postsynaptic location and release by stress. Neuroscience 126:533-540.

Chen Y, Rex CS, Rice CJ, Dubé CM, Gall CM, Lynch G, Baram TZ (2010) Correlated memory defects and hippocampal dendritic spine loss after acute stress involve corticotropin-releasing hormone signaling. Proc Natl Acad Sci U S A 107:13123-13128.

De Souza EB, Insel TR, Perrin MH, Rivier J, Vale WW, Kuhar MJ (1985) Corticotropin-releasing factor receptors are widely distributed within the rat central nervous system: an autoradiographic study. J Neurosci 5:3189-3203.

Hillhouse EW, Grammatopoulos DK (2006) The molecular mechanisms underlying the regulation of the biological activity of corticotropinreleasing hormone receptors: implications for physiology and pathophysiology. Endocr Rev 27:260-286

Ivy AS, Rex CS, Chen Y, Dub é C, Maras PM, Grigoriadis DE, Gall CM, Lynch G, Baram TZ (2010) Hippocampal dysfunction and cognitive impairments provoked by chronic earlylife stress involve excessive activation of $\mathrm{CRH}$ receptors. J Neurosci 30:13005-13015.

McEwen BS (1999) Stress and hippocampal plasticity. Annu Rev Neurosci 22:105-122.

Merchenthaler I (1984) Corticotropin releasing factor (CRF)-like immunoreactivity in the rat central nervous system. Extrahypothalamic distribution. Peptides 5 [Suppl 1]:53-69.

Radulovic J, Rühmann A, Liepold T, Spiess J (1999) Modulation of learning and anxiety by corticotropin-releasing factor (CRF) and stress: differential roles of CRF receptors 1 and 2. J Neurosci 19:5016-5025.

Rebaudo R, Melani R, Balestrino M, Izvarina N (2001) Electrophysiological effects of sustained delivery of CRF and its receptor agonists in hippocampal slices. Brain Res 922:112-117. 